\title{
Effective monitoring of diabetic patients on progressive chronic kidney disease among patients attending diabetic clinic at Muhimbili National Hospital, Tanzania
}

\author{
Gudila Valentine Shirima ${ }^{1}$, Paschal Joseph Rugajjo ${ }^{2}$, Obadia Venance Nyongole ${ }^{2,{ }^{*}}$ Francis Furia ${ }^{2}$ and Kimu \\ Marko Njiku ${ }^{3}$ \\ ${ }^{1}$ Nephrology Unit, Department of Pediatrics and Child Health, Muhimbili National Hospital, Dar es Salaam, Tanzania. \\ ${ }^{2}$ Schools of Medicine, Muhimbili University of Health and Allied Sciences, Dar es Salaam, Tanzania. \\ ${ }^{3}$ Department of Urology, Muhimbili National Hospital, Dar es Salaam, Tanzania.
}

Magna Scientia Advanced Research and Reviews, 2021, 01(03), 044-052

Publication history: Received on 02 February 2021; revised on 05 March 2021; accepted on 07 March 2021

Article DOI: https://doi.org/10.30574/msarr.2021.1.3.0025

\begin{abstract}
Background: Diabetic Kidney Disease is a complication of Diabetes Mellitus (DM) following the natural history of diabetes. Worldwide up to $40 \%$ of patients with diabetes mellitus will develop Diabetic Kidney Disease. Kidney Disease Outcome Quality Initiative (KDOQI) guidelines recommends monitoring for proteinuria, blood sugar, renal functions and blood pressure in the patients with DM so that progression to complications including renal failure is prevented. Our study aimed to audit on effective monitoring of progressive chronic kidney disease among patients attending diabetic clinic at Muhimbili National Hospital.
\end{abstract}

Material and methods: This was a retrospective clinical audit which included patients who attended diabetic clinic at Muhimbili National Hospital in 1st January 2017 to 31st December 2017. Simple random sampling technique using software called OpenEpi Random Program was used to get the sample of 120 patients. Patients with missing clinical notes were excluded from the audit. Records of the clinical notes, socio-demographic characteristics and investigations of the selected patients were retrieved from the Jeeva system and recorded using a structured questionnaire. We analyzed our data using Statistical Package for Social Sciences (SPSS) version 20.0

Results: One hundred and fourteen DM patents records were reviewed. Around $79 \%$ of the patients involved in the study were aged above 45years, 60\% were females. Majority had type 2 DM (76.3\%). Minority i.e. 15/114 (13.2\%) of the patients had their urine for protein checked in the year 2017 as well as serum creatinine in the last 3 months. Seven percent had HbA1c test done i.e. 8/114 (7\%). Blood pressure was monitored in 72.8\% (83/114) of the patients. Only16 out of 66 patients $(24.2 \%)$ had blood pressure controlled. Majority of the patients 10/15 (66.7\%) who had renal functions records had normal eGFR. Findings on monitoring for proteinuria, renal functions, control of HbA1c and Blood pressure control were all below audit standards.

Conclusion: Our study found that there is generally poor monitoring of diabetic outpatient for progressive chronic kidney disease when compared to the KDOQI standards which calls the health care providers to adhere on set SOPs according to the guidelines available aiming at improving services and quality of life to diabetic patient.

Keywords: Diabetic Kidney Disease; Progressive Chronic Kidney Disease

\footnotetext{
${ }^{*}$ Corresponding author: Obadia Venance Nyongole

Schools of Medicine, Muhimbili University of Health and Allied Sciences, Dar es Salaam, Tanzania. 


\section{Background and literature review}

Diabetic kidney disease (DKD) is a clinical syndrome characterized by a progressive loss of kidney function resulting from diabetes. Diabetic kidney disease (DKD) was previously known as diabetic nephropathy and is defined as diabetes with albuminuria (ratio of urine albumin to creatinine $\geq 30 \mathrm{mg} / \mathrm{g}$ ), impaired glomerular filtration rate $(<60$ $\mathrm{ml} / \mathrm{min} / 1.73 \mathrm{~m} 2$ ), or both and is the single strongest predictor of mortality in patients with diabetes [1].There is an increased risk of Diabetic nephropathy when the microalbuminuria persists and progress to proteinuria $(>300 \mathrm{mg} /$ day) and usually occurs around 15years from the onset of the disease. Hypertension and CKD development set in with progressive increase in proteinuria. There are data regarding natural history of type $1 \mathrm{DM}$. In type 2DM, CKD can result from macrovascular disease i.e. without microalbuminuria [2-5].

\section{Natural History of Type 1 Diabetic Nephropathy}

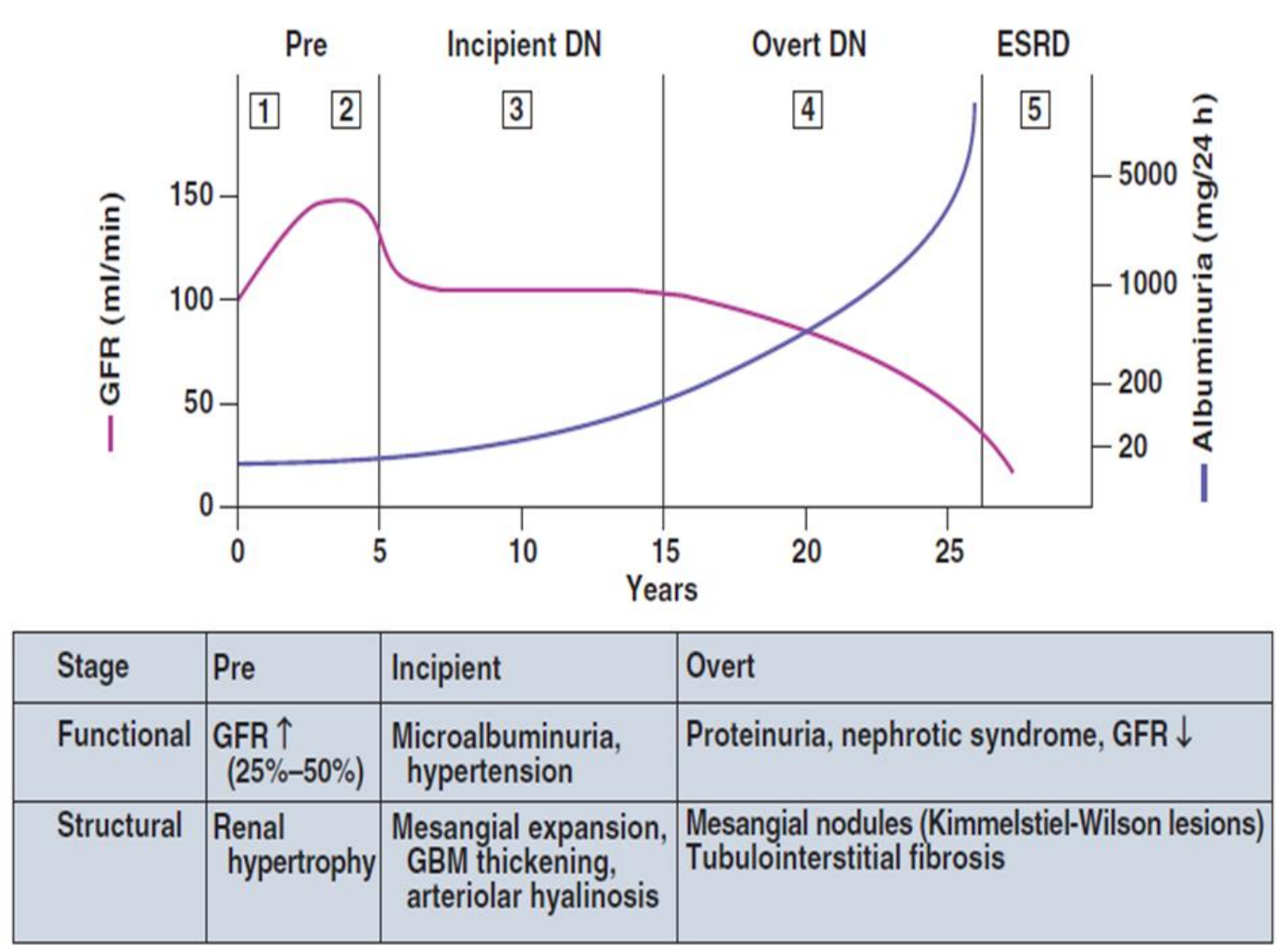

Figure 1 Natural history of type 1 diabetic Nephropathy (DN). Functional and structural manifestation of DN. Number 1 to 5 are the stages of DN as described by Mogensen ${ }^{2}$

Diabetes contributes to up to $40-50 \%$ of CKD patients. ESRD secondary to diabetes is preventable. Early CKD stages have been associated with detection of albumin in urine. After a period of approximately 10years of the onset DM Type $1,10-28 \%$ of the patients will develop microalbuminuria. The increased prevalence of diabetes has led to an increase in the number of macro-vascular) such as coronary heart disease, stroke) and microvascular complications of diabetes (such visual impairment, diabetic kidney disease (DKD), and end stage renal disease (ESRD)). However, diabetes remains the most common reason for progression to ESRD in the US and in many parts of the world [6-8].

It is estimated that in the next 5-10years 25 to $40 \%$ of those developing microalbuminuria will progress to overt macroalbuminuria. United Kingdom Prospective Diabetes Study (UKPDS) and the Diabetes Control and Complications Trial (DCCT), have shown that poor glycemic control is associated with an increased risk of developing nephropathy (microalbuminuria) and other microvascular complications of diabetes, such as retinopathy and neuropathy. The risk is increased for all HbA1C levels above the non-diabetic range $(<6.5 \%)$, and greatest at levels $>12 \%$.UKPDS showed that 
reducing the HbA1c level by approximately $0.9 \%$ in patients with type 2 diabetes reduces the risk for development of micro-vascular complications, including nephropathy[9].

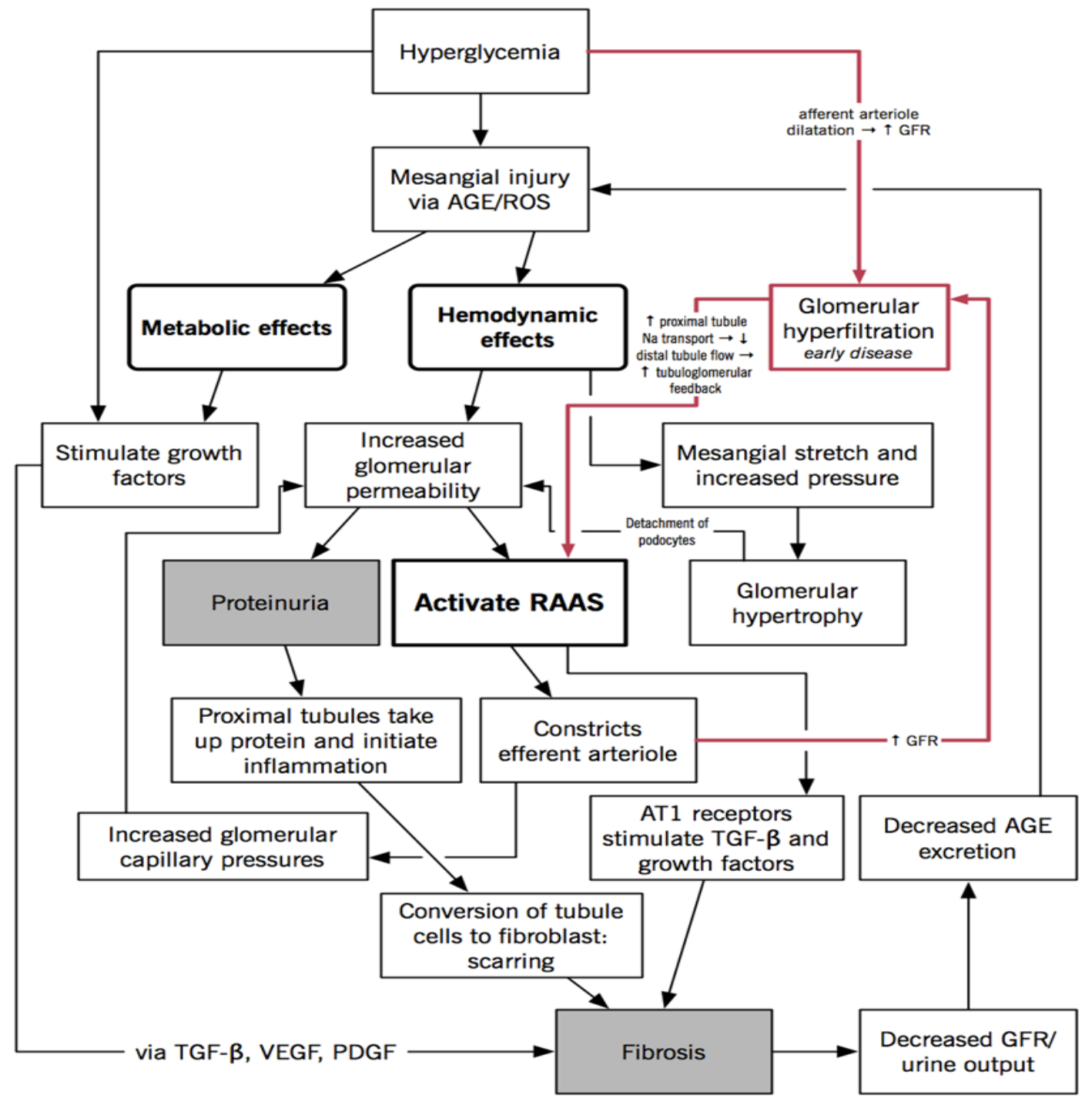

Figure 2 Pathophysiology of diabetic nephropathy involves interaction between metabolic and hemodynamic factors.

The Hypertension and Diabetes Study (HDS) and Hass Lacher C. et al; showed that 40-70\% of type 2 and $25 \%$ of type 1 DM patient will develop hypertension respectively. Decline of GFR will correlate with increase in blood pressure. Raised blood pressure in diabetic patients has also been found to be risk factor for both macro-vascular and micro-vascular complications including diabetic nephropathy [10-11].

Monitoring patients with DM for progression of DKD guides on the intervention and therefore crucial for the prevention of ESRD. Kidney Disease Outcome Quality Initiative (KDOQI) guidelines for diabetes and CKD update 2012 recommends the following regarding monitoring of patients with $\mathrm{DM}(\mathrm{HbA} 1 \mathrm{C}$ should be less than $7 \%$ to prevent progression of CKD and should be monitored three monthly,Should be monitored for microalbuminuria at least yearly,Blood pressure should be $<130 / 80 \mathrm{mmHg}$ for nonproteinuric and $125 / 75 \mathrm{mmHg}$ for proteinuric patients, Should be monitored for renal functions three monthly,use of ACE-I or/and ARB in normotensive/ hypertensive patients with diabetes and albuminuria levels $>30 \mathrm{mg} / \mathrm{g}$ who are at high risk of CKD or its progression,Should be referred to nephrologists when eGFR is $<60 \mathrm{ml} / \mathrm{ml} / 1.73 \mathrm{~m} 2$ ). We aimed to audit on effective monitoring of diabetic patients for progressive chronic kidney disease among patients attending diabetic clinic at MNH from January 2017 to December 2017.

Material and methods: This was a retrospective clinical audit which included patients who attended diabetic clinic at Muhimbili National Hospital in 1st January 2017 to 31st December 2017. 


\subsection{Data collection}

Through computerized information system, and by assistance from medical recorders, patients who have been at the clinic from 1st January 2017 to 31st January 2017 were identified. The patients' information was then gathered through Jeeva system from January 2017 to December 2017. Patients whose clinical notes were missing and those who did not attend clinic in last 3months (September to December 2017) were excluded from the audit. Estimated glomerular filtration rate was calculated by the MDRD formula for those who had records on serum creatinine.

\subsection{Sample size}

Simple random sampling technique using software called OpenEpi Random Program was used to get the convenient sample of 120 patients.

\subsection{Data entry and analysis}

The entry and analysis of audit data was done using statistical package for social sciences (SPSS) version 20.0. Continuous data were grouped and together with categorical data were presented as percentages using frequency distribution tables.

\subsection{Ethical clearance}

Proposal was presented to the department of internal medicine at MUHAS for approval. The head of unit in endocrinology at MNH was informed of the audit after getting permission of conducting this audit from the MNH administration.

\section{Results}

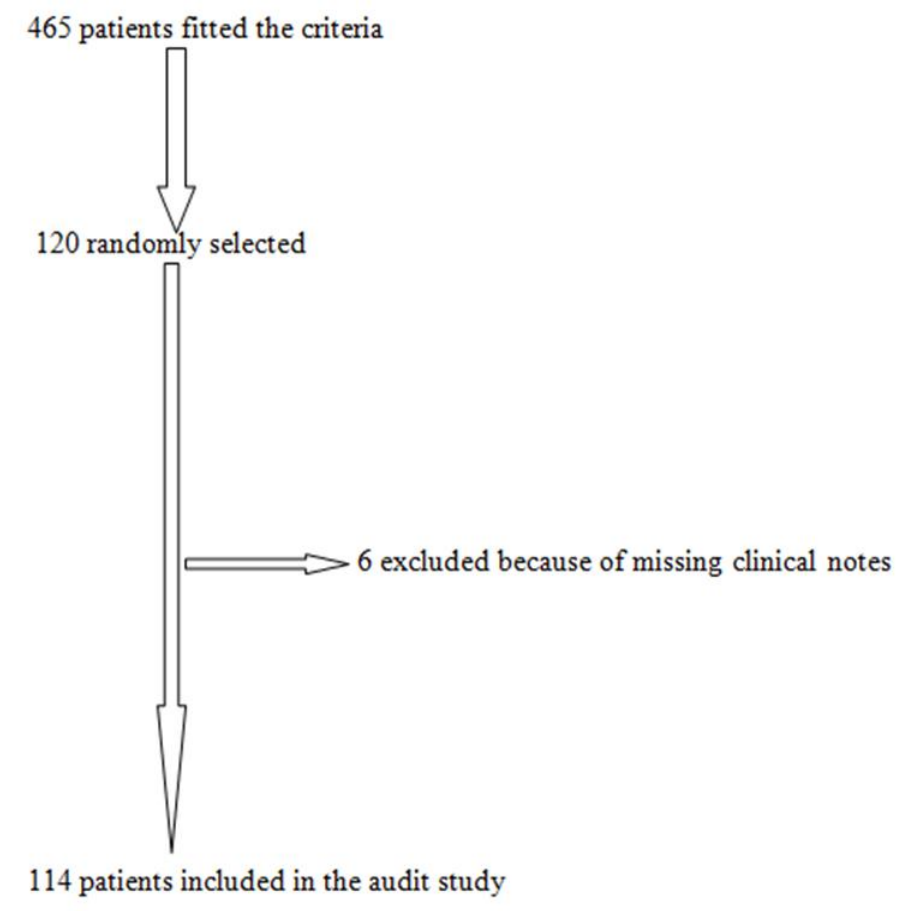

Figure 3 Flow Chart

Approximately $79 \%$ of the patients involved in the audit were aged above $45 y e a r s, 60 \%$ were females. Majority had type 2 DM (76.3\%). The duration of illness was documented only in $26 \%$ of participants, $50 \%$ of those with documented duration were diagnosed 10 or more years back. 
Table 1 Showing socio-demographic and clinical characteristics of the patients attending diabetic clinic ( $\mathrm{N}=114)$.

\begin{tabular}{|c|c|}
\hline Characteristics of the patients & Frequency n (\%) \\
\hline \multicolumn{2}{|l|}{ Age (years) } \\
\hline 18-30 & $12(10.5 \%)$ \\
\hline $31-45$ & $12(10.5 \%)$ \\
\hline $46-60$ & $52(45.6 \%)$ \\
\hline 61 and above & $38(33.3 \%)$ \\
\hline \multicolumn{2}{|l|}{ Sex } \\
\hline Male & $46(40.4 \%)$ \\
\hline Female & $68(59.6 \%)$ \\
\hline \multicolumn{2}{|l|}{ DM type } \\
\hline 1 & $11(9.6 \%)$ \\
\hline 2 & $87(76.3 \%)$ \\
\hline Not documented & $16(14 \%)$ \\
\hline \multicolumn{2}{|l|}{ Duration of illness (years) } \\
\hline$<5$ & $9(7.9 \%)$ \\
\hline $5-9$ & $6(5.3 \%)$ \\
\hline 10 or more & $15(13.2 \%)$ \\
\hline Not documented & $84(73.4 \%)$ \\
\hline \multicolumn{2}{|c|}{ BP measured ever in the year 2017} \\
\hline Yes & $83(72.8 \%)$ \\
\hline No & $31(27.2 \%)$ \\
\hline
\end{tabular}

Table 2 Showing whether level of proteinuria, creatinine and HbA1c were monitored (N=114).

\begin{tabular}{|c|c|}
\hline Parameter & Frequency n (\%) \\
\hline \multicolumn{2}{|c|}{ Level of protein in urine yearly } \\
\hline Yes & $15(13.2 \%)$ \\
\hline No & $99(86.8 \%)$ \\
\hline \multicolumn{2}{|c|}{ Serum creatinine ever in the year } \\
\hline Yes & $38(33.3 \%)$ \\
\hline No & $76(66.7 \%)$ \\
\hline \multicolumn{2}{|c|}{ At least 3BP records in a year } \\
\hline Yes & $83(72.8 \%)$ \\
\hline No & $31(27.2 \%)$ \\
\hline \multicolumn{2}{|c|}{$\mathrm{BP}$ recorded in the last 3 months } \\
\hline Yes & $66(57.9 \%)$ \\
\hline No & $48(42.1 \%)$ \\
\hline \multicolumn{2}{|c|}{ Serum creatinine in the last 3months } \\
\hline Yes & $15(13.2 \%)$ \\
\hline No & $99(86.8 \%)$ \\
\hline \multicolumn{2}{|c|}{ HbA1c in the last 3 months } \\
\hline Yes & $8(7 \%)$ \\
\hline No & $106(93 \%)$ \\
\hline
\end{tabular}


Minority i.e. 15/114 (13.2\%) of the patients had their urine for protein checked in the year 2017 as well as serum creatinine in the last 3months. Very few had been tested for HbA1c (7\%). Most of the patients had their blood pressure monitored $(72.8 \%)$.

Table 3 Control of Blood pressure, HbA1c and eGFR levels during the year 2017 among patients who had records.

\begin{tabular}{|l|l|}
\hline Parameter & Frequency n (\%) \\
\hline Average BP in the year (mmHg) n=83 & \\
\hline$<130 / 80$ & $25(30.1 \%)$ \\
\hline$>130 / 80$ & $58(69.9 \%)$ \\
\hline Total & $83(100 \%)$ \\
\hline BP in the last 3months (mmHg) n=66 & \\
\hline$<130 / 80$ & $16(24.2 \%)$ \\
\hline$>130 / 80$ & $50(75.8 \%)$ \\
\hline Total & $66(100 \%)$ \\
\hline HbA1c level in the last 3months (\%) $\mathrm{n}=8$ & \\
\hline$<7$ & $8(100 \%)$ \\
\hline $7-10$ & $0(0 \%)$ \\
\hline$>10$ & $0(0 \%)$ \\
\hline Total & $8(100 \%)$ \\
\hline eGFR in the last 3months (mls/min/1.73m2) n=15 & \\
\hline$>90$ & $10(66.7 \%)$ \\
\hline $60-89$ & $3(20 \%)$ \\
\hline $30-59$ & $1(6.7 \%)$ \\
\hline $15-29$ & $0(0 \%)$ \\
\hline$<15$ & $1(6.7 \%)$ \\
\hline Total & $15(100 \%)$ \\
\hline
\end{tabular}

Only16 out of 66 patients (24.2\%) had their blood pressure controlled. All of those tested for glycemic control had HbA1C $<7 \%$. Majority of the patients 10/15 (66.7\%) who had renal functions records had normal eGFR. None of the patients had calculated eGFR record.

\section{Discussion}

Diabetes mellitus has been described to be increasing worldwide but even more in developing countries, Tanzania included. Increasing age, duration of illness and female gender are some of the factors that have been found to be associated with diabetes mellitus [3, 12-13]. This clinical audit revealed similar findings where about $79 \%$ of the patients were aged above 45years and $60 \%$ were females. It's well known that duration of illness in DM contributes to development of diabetic nephropathy. In this current audit, majority (73.4\%) of the patient lack documentation of this important information.

In our study, we reviewed 114 case notes of DM patients in which performance was poor compared to the set standards in all assessed parameters. Majority had type 2 DM (76.3\%). The duration of illness was documented only in $26 \%$ of participants, $50 \%$ of those with documented duration were diagnosed 10 or more years back. Very few had been tested for HbA1c in the last 3months of the year 2017 i.e. 8/114 (7\%). All of those tested for glycemic control had HbA1C <7\%. Minority i.e. 15/114 (13.2\%) of the patients had their urine for protein checked in the year 2017. Blood pressure was 
monitored in $72.8 \%$ (83/114) of the patients. Only16 out of 66 patients $(24.2 \%)$ had blood pressure controlled Serum creatinine in the last 3months was monitored in 15/114 (13.2\%). Majority of the patients 10/15 (66.7\%) who had renal functions records had normal eGFR. None of the patients had calculated eGFR record.

In the clinical audit which was done in Nigeria among diabetic patients, 88.1\% were hypertensive. The eGFR ranged from 11 to $205 \mathrm{ml} / \mathrm{min} / 1.73 \mathrm{~m} 2,37.6 \%$ of the patients had eGFR less than $60 \mathrm{ml} / \mathrm{min} / 1.73 \mathrm{~m} 2$ and $22.3 \% \mathrm{had}$ proteinuria. Approximately $93 \%$ and $97 \%$ of the patients had their blood pressure records on the first and last follow up visits respectively. Less than half of the patients had record of serum electrolytes, urea, creatinine (41.9\%), urinalysis $(45.5 \%$ ) and packed cell volume while BP was recorded in $100 \%$ on their follow up files. None of the patients had records of HbA1C or eGFR[12].Mubarakali et al, did a cross-sectional study which was done in the northern-west part of Tanzania reported that $83.7 \%$ of diabetic patients had CKD; $80 \%$ had significant albuminuria and $24.7 \%$ had eGFR $<60 \mathrm{ml} / \mathrm{min}$. None of these patients were aware of their renal disease, and only $1.3 \%$ had a diagnosis of diabetic nephropathy recorded in their files [13].

Majority of our participants had type 2 DM (76.3\%) which was consistent with the findings of the studies done elsewhere [12]. A study from the United States in 2000 showed that almost $40 \%$ of type 2 DM patients have CKD to some degree [3].

Very few had been tested for HbA1c (7\%) in the current audit, which is lower than both the audit set goal of $20 \%$ and $90 \%$ of KDOQI guidelines. Although poor compared to the set standard, it is better than Nigerian finding of $0 \%$. UKPDS and the DCCT have shown that poor glycemic control is associated with an increased risk of developing micro albuminuria [9,22]. Lacking records on glycemic control put these patients at risk developing DKD even more. It's recommended, by KDOQI and the DCCT study that HbA1c kept below 7\% consistent with the findings of those with records of blood sugar in the current audit $[17,22]$. All of the patients with records were in private category (finding not shown in the results). Does this tell us its the cost issue? Or maybe those who are knowledgeable of the importance of the test are the once doing the test. Of note, before June 2016 the centre i.e. Muhimbili National Hospital had no HbA1c test; can it be that the health care providers are not aware of its availability? These need to be researched.

Fifteen patients (13.2\%) had their urine for protein checked at least once a year in this audit study. This was lower compared to this audit set standard 50\% and KDOQI guideline of $90 \%$. This audit finding was also lower compared to $45.8 \%$ in the similar audit done in Nigeria ${ }^{12}$. None of these two audits had microalbuminuria done. Another study done within the country had shown that $80 \%$ of DM patients presented with moderate to severely increased albuminuria ${ }^{13}$. The availability of the albusticks/urine protein dipsticks at the clinic, lack of patients' awareness on the importance, and even health care provider motivation could explain the low rates in monitoring. Tanzania being a third world country, cost could also be a reason but in this study there was no statistical difference between private versus cost sharing category of patients in terms of checking for proteinuria (information not shown in the results).

The study done on hypertension and diabetes study (HDS) and Hass Lacher C. et al; showed that 40-70\% of type 2 and $25 \%$ of type 1 DM patient will develop hypertension respectively. Most of the patients had their blood pressure monitored in the current audit (72.8\%) and this was consistent with other studies. Despite most of them being monitored for BP, only $24.2 \%$ had BP controlled which is lower than both audit set standard of $50 \%$ and KDOQI standard of $80 \%$, therefore poor performance [10-12].

The KDOQI recommends monitoring of renal functions every three months. ${ }^{17}$ In the cross-sectional study done by Mubarakali et al, $24.7 \%$ of DM patients had eGFR of $<60 \mathrm{mls} / \mathrm{min} / 1.73 \mathrm{~m} 2$ but only $1.3 \%$ had records on their files. In Nigerian study $41.9 \%$ had records of creatinine but in our case $33.3 \%$ was ever done in a year. This clinical audit revealed only (13.2\%) were checked creatinine in the latest 3months which was lower than both audit set standard of $30 \%$ and KDOQI standard of $90 \%$. Nevertheless, both studies had no records on eGFR. Calculation from the latest creatinine by MDRD formula revealed $13.4 \%$ had eGFR $<60 \mathrm{ml} / \mathrm{min} / 1.73 \mathrm{~m} 2$ in the current audit. This results are consistent with other studies where developed countries have reported screening for DN in $>70 \%$ of the patients and as poor in developing countries [19-21].

\section{Conclusion}

Monitoring for diabetic nephropathy among patients attending diabetic clinic at MNH clinic is poor. We need to develop guidelines/SOPs/protocols and use them accordingly. Also we need holistic approach in monitoring and managing patients with diabetes in prevention of DKD according to KDOQI guidelines. 


\section{Compliance with ethical standards}

\section{Acknowledgments}

We are thankful to all staff at MNH who assisted us during preparations of this work the hospital management for funding the study.

\section{Disclosure of conflict of interest}

The authors declare no competing interests.

\section{Authors' contributions}

GVS designed the study, collected data, performed data analysis and wrote the report with a manuscript. OVN participated in the study design and manuscript preparation. GS participated in the study design. PJR participated in the study design. NK participated in the study design.

\section{Statement of informed consent}

We reviewed patient case notes without identifiers having received permission from Muhimbili National Hospital.

\section{References}

[1] Reidy K, Kang H, Hostetter T, Susztak K. Molecular mechanisms of diabetic kidney disease, Journal of Clinical Investigation. 2014; 124(6): 2333-2340.

[2] Mogensen CE. How to protect the kidney in diabetic patients with special reference to NIDDM. Diabetes. 1997; 56(suppl 2): 104-111.

[3] Yokoyama et al. Higher incidence of diabetic nephropathy in type 2 than in type 1DM in early onset DM in Japan. Kidney int. 2000; 58: 302-11.

[4] Balard DJ et al. Epidermiology of persistent proteinuria in type 2 DM. Population based study in Rodester minresotu DM. 1988; 37: 405-12.

[5] Anderson A Ret al. Diabetic nephropathy in type 1(insulin dependent) diabetes: An epidermiology diabetologia. 1983; 25: 496-501.

[6] USRDS: The United States renal data system, American Journal of Kidney Diseases. 2003; 42(6): 1-230.

[7] E Ritz, I Rychlik, F Locatelli, S Halimi. End-stage renal failure in type 2 diabetes: a medical catastrophe of worldwide dimensions, American Journal of Kidney Diseases. 1999; 34(5): 795-808.

[8] AT Reutens. Epidemiology of Diabetic Kidney Disease, Medical Clinics of North America. 2013; 97(1): 1-18.

[9] UK Prospective Diabetes Study (UKPDS) Group. Intensive blood-glucose control with sulphonylureas or insulin compared with conventional treatment and risk of complications in patients with type 2 diabetes (UKPDS 33). Lancet. 1998; 352: 837-853.

[10] Hypertension on diabetes study (HDS). 1. Prevalence of hypertension in newly presenting type 2 diabetic patients and the association risk factors for cardiovascular and diabetes complications. J. Hypertension 1993; 11: 309-17.

[11] Hass Lacher C, Stech W, Wahl P, Ritz E. Blood pressure and metabolic control as risk factors for nephropathy in type 1 (insulin dependent) Diabetes. Diabetologia. 1985; 28: 6-11.

[12] Okafor UH, Ezeala A, Aneke E. Audit of Screening for Diabetic Nephropathy in a Teaching Hospital in Nigeria. J Diabetes Metab. 2015; 6: 525.

[13] Mubarakali N J, Samuel E Kalluvya SE , Mueller A ,Kabangila R, Smart LR, Downs JA, Peck RN. Prevalence of chronic kidney disease in diabetic adult out-patients in Tanzania. BMC Nephrology 2013, 14:183.

[14] Caramori ML, Fioretto P, Mauer M. The need for early predictors of diabetic nephropathy risk: is albumin excretion rate sufficient? Diabetes. 2000; 49: 1399-1408.

[15] Rossing P, Hommel E, Smidit UM, Parving HH. Impact of arterial blood pressureand albuminuria on progression of DN in IDDM patients.Diabetes. 1993; 42: 715-19. 
[16] Viberti GC, Laffel L, Gans DJ. Secondary prevention of Diabetic Nephropathy by captopril in patients with IDDM and microalbuminuria.J Am Soc Nephrol. 1994.

[17] KDOQI (Kidney Disease Outcome Quality Initiative) guidelines for diabetes and CKD update. 2012.

[18] Farnkrist LM, Lundman BM. Outcome of Diabetes care: Population based study. Int J Qual Health care. 2003; 15 : 301-307.

[19] Khunti K, Ganguli Set al. Features of primary care associated with variation with processand outcome of care of people with diabetes.Br J. Gen Pract. 2001; 51: 356-360.

[20] De Lusignan S, Chan T, Stevens P, et al. Identifying patients with chronic kidney disease from general practice computer records. Fam Pract. 2005;22(3):234-241.

[21] Diabetes control and complication trial research group.the effect of intensive treatment of diabetes in the development and progression of long term complication in insulin dependent diabetes mellitus.N Engl J Med.1993; 329: 977-986. 